Article

\title{
Enhanced Stability and Driving Performance of GO-Ag-NW-based Ionic Electroactive Polymer Actuators with Triton X-100-PEDOT:PSS Nanofibrils
}

\author{
Minjeong Park ${ }^{1}{ }^{\circledR}$, Seokju Yoo ${ }^{1}$, Yunkyeong Bae ${ }^{1}$, Seonpil Kim ${ }^{2}$ and Minhyon Jeon ${ }^{1, *}$ \\ 1 Department of Nanoscience and Engineering, Center for Nano Manufacturing, Inje University, \\ Gimhae 50834, Korea; mjpark9121@gmail.com (M.P.); yuinjae7@gmail.com (S.Y.); \\ yunkyeong.b@gmail.com (Y.B.) \\ 2 Department of Military Information Science, Gyeongju university, Gyeongju 38065, Korea; seonpil@gu.ac.kr \\ * Correspondence: mjeon@inje.ac.kr; Tel.: +82-55-320-3672; Fax: +82-55-320-3963
}

Received: 25 April 2019; Accepted: 16 May 2019; Published: 19 May 2019

\begin{abstract}
Ionic electroactive polymers (IEAPs) have received considerable attention for their flexibility, lightweight composition, large displacement, and low-voltage activation. Recently, many metal-nonmetal composite electrodes have been actively studied. Specifically, graphene oxide-silver nanowire (GO-Ag NW) composite electrodes offer advantages among IEAPs with metal-nonmetal composite electrodes. However, GO-Ag NW composite electrodes still show a decrease in displacement owing to low stability and durability during driving. Therefore, the durability and stability of the IEAPs with metal-nonmetal composite electrodes must be improved. One way to improve the device durability is coating the electrode surface with a protective layer. This layer must have enough flexibility and suitable electrical properties such that it does not hinder the IEAPs' driving. Herein, a poly(3,4-ethylenedioxythiophene)-poly(styrenesulfonate) (PEDOT:PSS) protective layer and 4-(1,1,3,3-tetramethylbutyl)phenyl-polyethylene glycol (Triton X-100) are applied to improve driving performance. Triton X-100 is a nonionic surfactant that transforms the PEDOT:PSS capsule into a nanofibril structure. In this study, a mixed Triton X-100/PEDOT:PSS protective layer at an optimum weight ratio was coated onto the GO-Ag NW composite-electrode-based IEAPs under various conditions. The IEAP actuators based on GO-Ag NW composite electrodes with a protective layer of PEDOT:PSS treated with Triton X-100 showed the best stability and durability.
\end{abstract}

Keywords: graphene oxide; silver nanowires; ionic electroactive polymer; poly(3,4ethylenedioxythiophene)-poly(styrenesulfonate) (PEDOT:PSS); 4-(1,1,3,3-Tetramethylbutyl)phenylpolyethylene glycol

\section{Introduction}

Ionic electroactive polymers (IEAPs) are among the most functional materials-based actuators. IEAPs have useful properties, such as a lightweight composition, a large working displacement under a low driving voltage, and a high energy density [1]. Electrodes are an important component of IEAPs. When sufficient, they provide high electrical conductivity, mechanical durability, and a smooth surface morphology [2]. The electrodes of IEAPs are categorized as metallic or nonmetallic. Noble metals (e.g., platinum, gold) with high electrical conductivity and electrochemical stability are often used as the metallic electrode of IEAPs. However, most metallic electrodes exhibit microcracks on the electrode surface, thus diminishing their surface electrical conductivity during long-term actuation [3,4]. Meanwhile, most nonmetallic electrodes are fabricated using transition metal oxides or carbon materials [1,5-9]. These materials can be assembled into electrodes via a physical hot-pressing method, which is simpler and faster than other fabrication methods $[10,11]$. However, nonmetallic 
electrodes are less conductive than metallic electrodes. To resolve such challenges, recent studies have increasingly investigated metallic-nonmetallic composite electrodes. Composite electrodes can solve both the occurrence of microcracks on the metallic electrode surface during driving and the low electrical conductivity of nonmetallic electrodes [12]. However, composite electrodes have a high contact resistance on their surfaces, which can instigate electrode burn out and decrease electrode durability during actuation.

Poly(3,4-ethylenedioxythiophene)-poly(styrenesulfonate) (PEDOT:PSS), a conductive polymer, is applied in various fields for its advantages of electrical conductivity and transparency. Particularly, PEDOT:PSS has been actively studied as a protective layer coated onto electrode materials such as carbon nanotubes (CNTs), graphene, and metal nanowires (NWs) with high contact resistance [13-16]. PEDOT:PSS is composed of a PEDOT phase and a PSS phase. The PEDOT phase is electrically conductive, but it is not suitable for solution processing because of its low solubility. On the other hand, the PSS phase is electrically insulating and water soluble. Because of these combined properties, the PEDOT:PSS is both electrically conductive and water soluble. However, the PEDOT phase's tendency to aggregate can make electrodes brittle.

Triton X-100 (4-(1,1,3,3-tetramethylbutyl)phenyl-polyethylene glycol) is composed of hydrophilic polyethylene oxide and hydrophobic 4-(1,1,3,3-tetramethylbutyl)-phenyl. This composition imparts it with amphiphilic properties owing to its hydrophilic "head" and hydrophobic "tail" and is thus a nonionic surfactant. It is widely used for mixing polar and nonpolar materials or lysing cells to extract proteins or organelles in biological fields through its surfactant property [17]. Moreover, Triton X-100 can lead to the formation of PEDOT nanofibrils in a viscoelastic medium due to its amphiphilic molecular structure of Triton X-100. Additionally, it can solve the PEDOT phase aggregation problem. Through this mechanism, the structure of PEDOT:PSS can be modified to have a flexible morphology. In addition, it enables improving the electrical conductivity by removing the PSS phase, which acts an insulator, through post-treatment [18-20].

In this study, Triton X-100-PEDOT:PSS-coated graphene oxide and silver NWs (TP/GO-Ag NWs) were fabricated as composite-electrode-based IEAPs. Herein, it is demonstrated that Triton X-100 can transform the aggregated structure of PEDOT:PSS into a nanofibril structure and thus improve electrical properties. Furthermore, the Triton X-100/PEDOT (TP) mixture coated on GO-Ag NW composite electrodes is used as a protective layer for the GO-Ag NW electrode. The improved characteristics of TP/GO-Ag-NW-based IEAPs were observed with a focus on electrical conductivity and driving properties.

\section{Materials and Methods}

\subsection{Materials}

PEDOT:PSS, Triton X-100, GO, and Ag NWs were used to fabricate the composite electrodes of the IEAPs. PEDOT:PSS (1.3 wt \%) and Triton X-100 were purchased from Sigma-Aldrich, St. Louis, MO, US. GO was synthesized using Hummer's method [21]. The Ag NW solution was purchased from Duksan Hi-Metal, Ulsan, Korea. Nafion 117 (N117) and 20 wt \% Nafion resin (Nafion solution) were purchased from the DuPont Company, Midland, MI, US. 1-Ethyl-3-methylimidazolium trifluoromethylsulfonate (EMIM-Otf), an ionic liquid (IL), was purchased from Merck KGaA, Darmstadt, Germany. A polyvinylidene difluoride (PVDF) membrane filter with a pore size of $0.20 \mu \mathrm{m}$ and a diameter $\emptyset 47 \mathrm{~mm}$ was purchased from Hyundai Micro., Ltd, Seongnam, Korea.

\subsection{Fabrication of IEAP Actuators Based on TP/GO-Ag NW Electrode}

GO powder $(330 \mathrm{mg})$ and deionized water $(5 \mathrm{~mL})$ were stirred at $250 \mathrm{rpm}$ for $5 \mathrm{~min}$ in a beaker to prepare the GO solution. The GO and Ag NW solutions were mixed in a 1:2.5 volume ratio. This GO-Ag NW mixture was used to fabricate a composite paper electrode using a vacuum filtration system. This paper electrode was dried at $100^{\circ} \mathrm{C}$ for $5 \mathrm{~min}$ in a vacuum oven. We performed ion 
exchange on the purchased Nafion following a previously-reported method [19]. The Nafion resin was painted directly onto both surfaces of the N117 membrane as an additive solution to paste the GO-Ag NW composite electrode to the Nafion membrane, forming an electrode/membrane/electrode structure. This structure was then hot-pressed at $0.1 \mathrm{MPa}$ and $100^{\circ} \mathrm{C}$ for $2 \mathrm{~min}$. Thus, we obtained IEAPs based on GO-Ag NW electrodes. In addition, Triton X-100 was mixed with PEDOT:PSS at various volume ratios and spin-coated onto both surfaces of the IEAP actuator based on the GO-Ag NW composite electrodes. Two coating conditions were considered: duration and speed. The PSS phases were then removed with methanol. Finally, IEAPs based on TP/GO-Ag NW composite electrodes were fabricated (width $\times$ length $($ Free length $) \times$ thickness $=5 \mathrm{~mm} \times 35 \mathrm{~mm}(30 \mathrm{~mm}) \times 200 \mu \mathrm{m})$.

\subsection{Characterization}

The transmittances of samples with different Triton X-100 weight ratios were investigated using an ultraviolet visible spectrophotometer (LAMBDA 465, PerkinElmer, Seoul, Korea). The morphologies and thickness of the electrodes were investigated using atomic force microscope (AFM; XE-100, Park Systems, Suwon, Korea) and field-emission scanning electron microscopy (FE-SEM; S-4300, Hitachi, Tokyo, Japan). The sheet resistances of electrodes were measured using a four-point probe (FPP-HS 8 , DASOL ENG, Cheongju, Korea). The driving characteristics of the actuators were measured using a laser displacement sensor (ZS-LD80, OMRON Korea, Seoul, Korea) in an actuation performance analyzer that we constructed.

\section{Results}

\subsection{Characterization of the TP/GO-Ag NW Electrode}

Triton X-100 and PEDOT:PSS were mixed at six different weight ratios $(0.0,1.0,2.5,5.0,7.5$, and $10.0 \mathrm{wt} \%$ Triton X-100). In addition, the PSS phases of all Triton X-100 and PEDOT:PSS mixtures were removed with methanol. In order to optimize the Triton X-100 concentration in the TP mixtures, protective layers were separately spin-coated on glass. Figure 1a shows the TP mixtures with different Triton X-100 contents coated on glass. Pure PEDOT:PSS did not uniformly coat the glass because of the high surface tension of PEDOT:PSS and its aggregated structure. However, the added Triton X-100 reduced the surface tension of the various TP mixtures. Thus, the TP mixtures uniformly coated the glass, in contrast with the pure PEDOT:PSS solution.
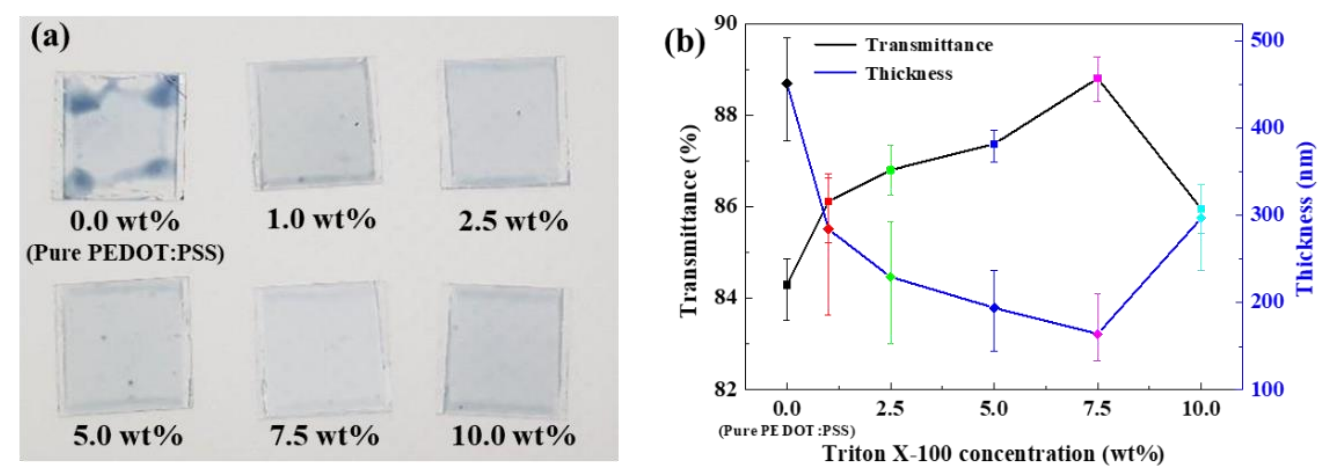

Figure 1. (a) The optical images of Triton X-100/poly(3,4-ethylenedioxythiophene) (TP) mixture coated on glass and (b) transmittance (black line) and thickness (blue line) of films with different Triton X-100 weight ratios.

Figure $1 \mathrm{~b}$ shows the optical transmittance at $550 \mathrm{~nm}$ and the thickness of the pure PEDOT:PSS and TP mixtures coated on the glass. The transmittance and thickness of all samples are inversely proportional. Pure PEDOT:PSS had lower transmittance (84.29\%) than the TP mixtures. Meanwhile, the TP mixture with $7.5 \mathrm{wt} \%$ Triton X-100 had the highest transmittance $(88.81 \%)$ and the thickest 
coating $(164.29 \mathrm{~nm})$ among the TP mixtures. These results suggests that the aggregated structure of PEDOT:PSS transformed into a nanofibril structure through the addition of Triton X-100.

Figure 2a-f shows AFM images of the 0.0, 1.0, 2.5, 5.0, 7.5, and $10.0 \mathrm{wt} \%$ TP layers, respectively, which reveal the surface roughness and structure of the TP layer. For accurate comparison, we scanned and analyzed an area of $5 \times 5 \mu \mathrm{m}^{2}$. These results reveal that the Triton X-100 treatment transformed the PEDOT:PSS aggregated structure to a nanofibril structure. Notably, the TP mixture with $7.5 \mathrm{wt} \%$ Triton X-100 had a nanofibril structure and lower surface roughness than the other TP mixtures, thus corroborating the transmittance and thickness results. At $10.0 \mathrm{wt} \%$, the of the TP mixture exhibited aggregation, meaning that the amount of Triton X100 exceeded the critical micelle concentration (CMC), thereby forming micelles. The CMC, the surfactant concentration above which micelles form, is an important characteristic of a surfactant. Specifically, after reaching the $\mathrm{CMC}$, any additional surfactants added to the system form micelles, which are large molecules formed by clusters of surfactant particles such as Triton X100. The size of the particles increases with increasing molecular aggregation. Figure $2 \mathrm{~g}$ schematically illustrates the mechanism underlying the PEDOT:PSS transformation with the Triton X100 treatment.
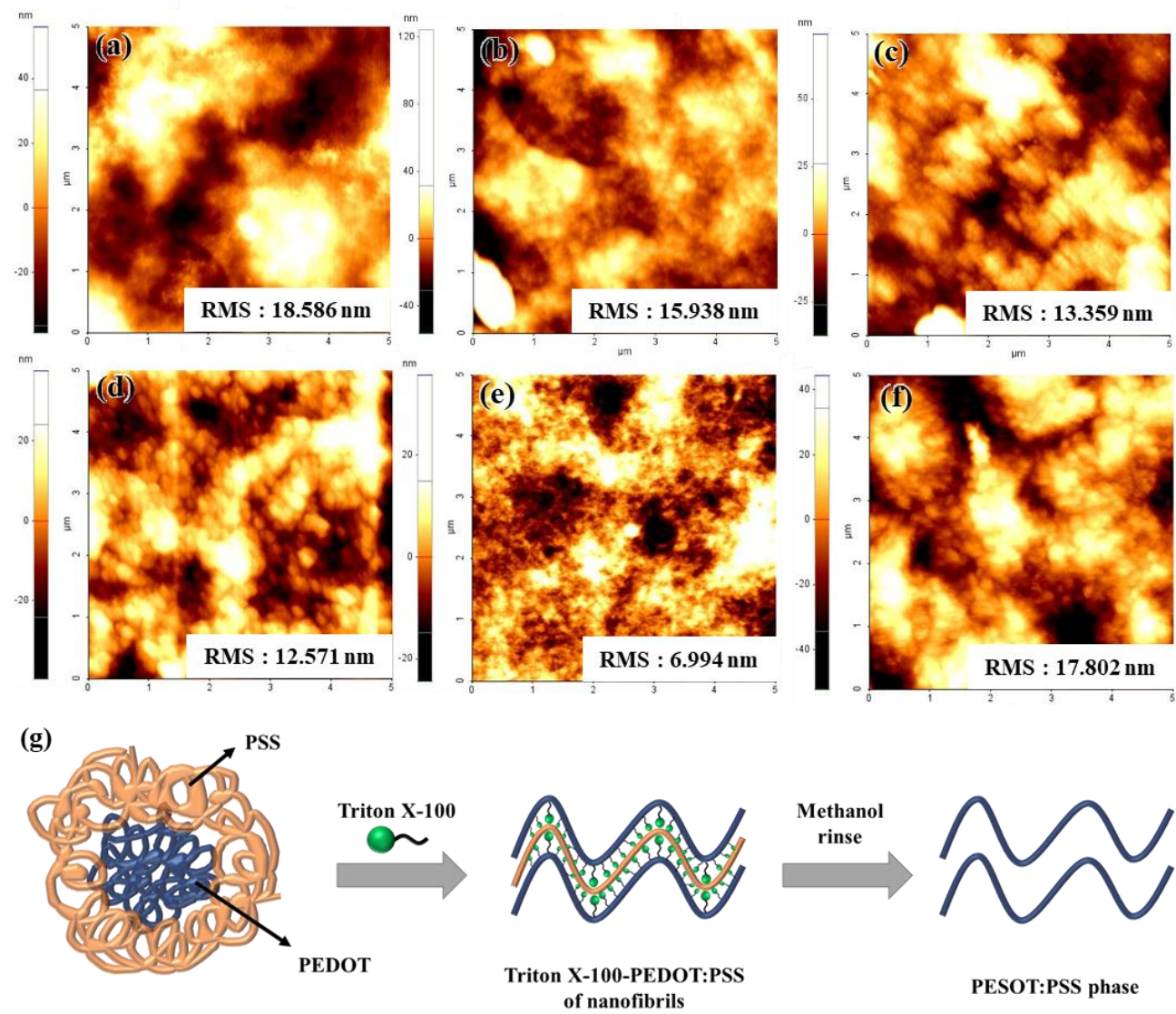

Figure 2. Atomic force microscope (AFM) images with a scanning area $5 \mu \mathrm{m} \times 5 \mu \mathrm{m}$ and root-mean-square (RMS) surface roughness values. (a) $0.0 \mathrm{wt} \%$, (b) $1.0 \mathrm{wt} \%$, (c) $2.5 \mathrm{wt} \%$, (d) $5.0 \mathrm{wt} \%$, (e) $7.5 \mathrm{wt} \%$, (f) $10.0 \mathrm{wt} \%$ Triton X100. (g) Schematic diagram illustrating the phase transition of poly(3,4-ethylenedioxythiophene)-poly(styrenesulfonate) (PEDOT:PSS) in the presence of Triton X-100.

Figure 3 graphically represents the sheet resistance of the GO-Ag NW composite electrodes coated with different TP mixtures, which was measured using a four-point probe. The TP-mixture-coated GO-Ag NW electrodes had a lower sheet resistance than the GO-Ag NW electrode without Triton $\mathrm{X}-100$. In addition, washing with methanol decreased the sheet resistances of all the TP-mixture-coated GO-Ag NW electrodes. Particularly, the $7.5 \mathrm{wt} \%$ Triton X-100 TP mixture had the lowest sheet 
resistance. Table 1 lists the sheet resistance of the samples in detail. Accordingly, the $7.5 \mathrm{wt} \%$ Triton $\mathrm{X}-100 \mathrm{TP}$ mixture is the most suitable choice as the protective layer for IEAP electrodes.

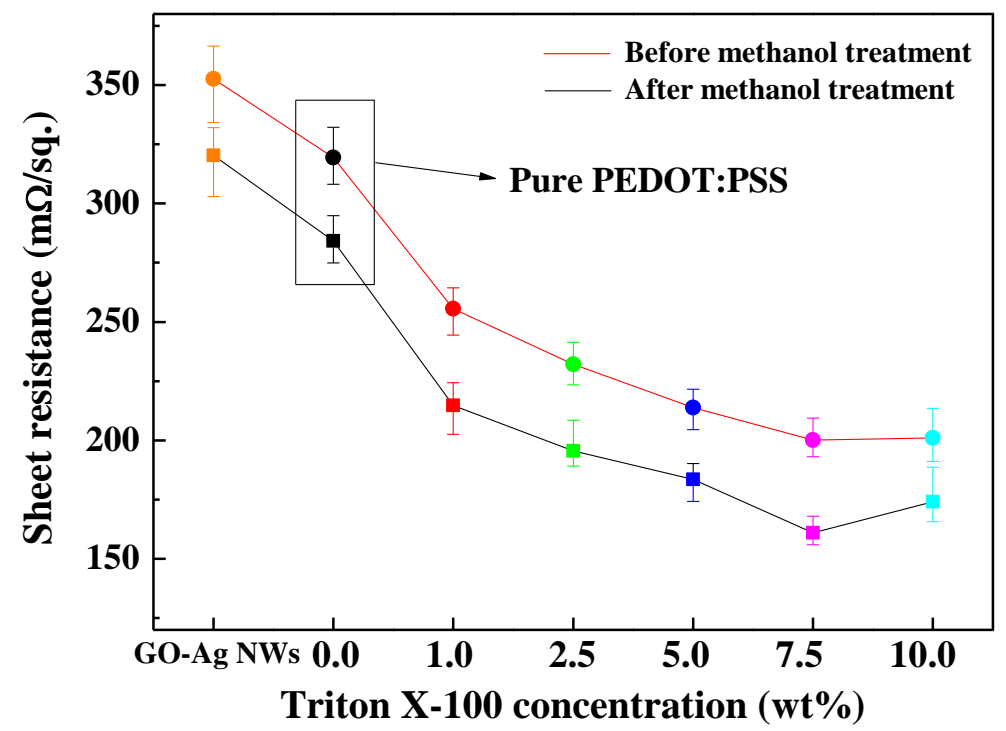

Figure 3. Sheet resistances before (red line) and after (black line) methanol treatment with various Triton X-100 weight ratios.

Table 1. Sheet resistances before and after methanol treatment for various Triton X-100 weight ratios.

\begin{tabular}{ccc}
\hline \multirow{2}{*}{ Samples } & \multicolumn{2}{c}{ Sheet Resistance $(\mathrm{m} \Omega / \mathbf{s q})}$. \\
\cline { 2 - 3 } & Before Methanol Treatment & After Methanol Treatment \\
\hline GO-Ag NWs & 352.65 & 320.25 \\
$0.0 \mathrm{wt} \%$ (Pure PEDOT:PSS) & 319.36 & 284.16 \\
$1.0 \mathrm{wt} \%$ Triton X-100 & 255.52 & 214.73 \\
$2.5 \mathrm{wt} \%$ Triton X-100 & 232.15 & 195.57 \\
$5.0 \mathrm{wt} \%$ Triton X-100 & 213.80 & 183.57 \\
$7.5 \mathrm{wt} \%$ Triton X-100 & 200.08 & 161.00 \\
$10.0 \mathrm{wt} \%$ Triton X-100 & 201.00 & 174.00 \\
\hline
\end{tabular}

Next, to optimize the spin coating conditions of the TP mixture with $7.5 \mathrm{wt} \%$ for the GO-Ag NW electrode, the TP/GO-Ag NW electrodes were fabricated with different coating times $(0,15,30$, and $45 \mathrm{~s}$ ) and coating speeds (300, 500, 700, 1000, and $2000 \mathrm{rpm})$. Figure 4 a shows the sheet resistance of the $\mathrm{TP} / \mathrm{GO}-\mathrm{Ag}$ NW electrodes with different coating times and a fixed coating speed of $1000 \mathrm{rpm}$. In this figure, the results for the TP-mixture-coated electrodes are compared with those of the GO-Ag NW electrode and the GO-Ag NW electrode coated with the pure PEDOT:PSS (P/GO-Ag NW electrode). The TP mixture with $7.5 \mathrm{wt} \%$ Triton X-100 coated for $30 \mathrm{~s}$ had a lower sheet resistance than the other samples. Figure $4 \mathrm{~b}$ shows the sheet resistance of the TP/GO-Ag NW electrode at different coating speeds (coating time: $30 \mathrm{~s}$ ). The $7.5 \mathrm{wt} \%$ Triton X-100 TP mixture coated at $1000 \mathrm{rpm}$ for $30 \mathrm{~s}$ provided the lowest sheet resistance of about $161 \mathrm{~m} \Omega / \mathrm{sq}$. Thus, the optimal spin coating conditions for coating the TP mixture on the GO-Ag NW electrode were $7.5 \mathrm{wt} \%$ Triton X100, a coating time of $30 \mathrm{~s}$, and a coating speed of $1000 \mathrm{rpm}$. The sheet resistance of this electrode was $49.73 \%$ and $82.10 \%$ lower than those of the GO-Ag NW electrode and the P/GO-Ag NW electrode, respectively. These optimized conditions were thus used for subsequent experiments. 

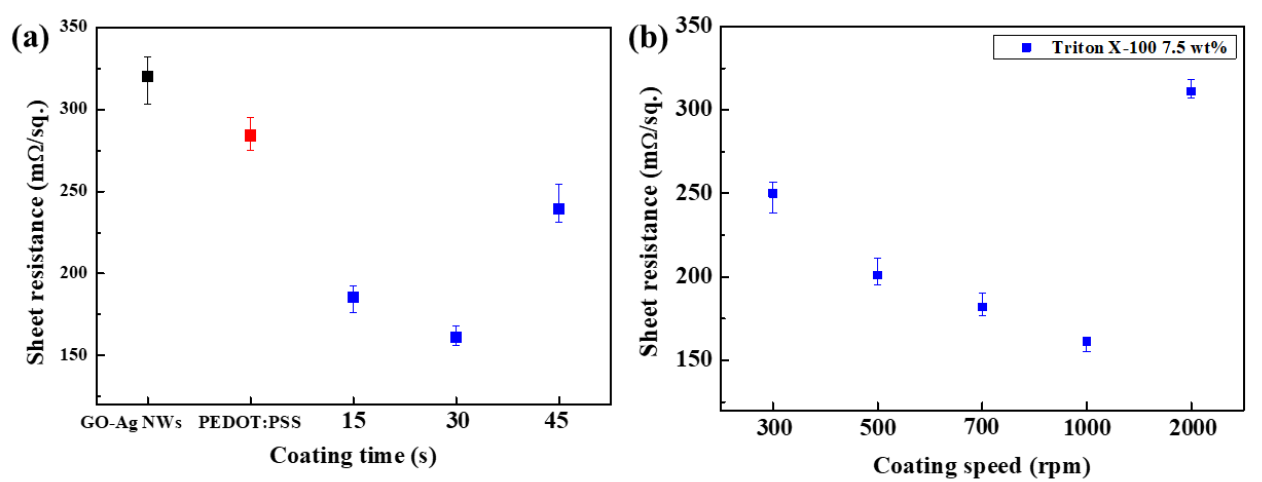

Figure 4. Sheet resistances of the (a) uncoated electrode (black point), P/GO-Ag NW electrode, and $7.5 \mathrm{wt} \%$ Triton X100 TP/GO-Ag NW electrodes coated for different coating times at $1000 \mathrm{rpm}$. (b) Sheet resistances of the $7.5 \mathrm{wt} \%$ Triton X100 TP/GO-Ag NW electrodes coated at different coating speeds.

\subsection{Actuation Performance of IEAP Actuators Based on TP/GO-Ag NW Electrode}

The driving performance of IEAP actuators with different electrodes was measured and observed, and the results are presented in Figure 5. The PEDOT:PSS-based IEAPs were measured to confirm the effect of $7.5 \mathrm{wt} \%$ Triton X-100. In order to investigate the effects of the electrode type on each actuator, the actuation performances of the IEAPs with three types of electrodes (GO-Ag NWs, P/GO-Ag NWs, and TP/GO-Ag NWs) were measured under $\pm 2.5 \mathrm{~V}_{\mathrm{AC}}$ and $0.2 \mathrm{~Hz}$, as shown in Figure $5 \mathrm{a}$,b. Figure $5 \mathrm{a}$ shows the harmonic responses of the three types of IEAPs. The TP/GO-Ag-NW-based IEAPs had larger tip displacements than the other IEAPs.
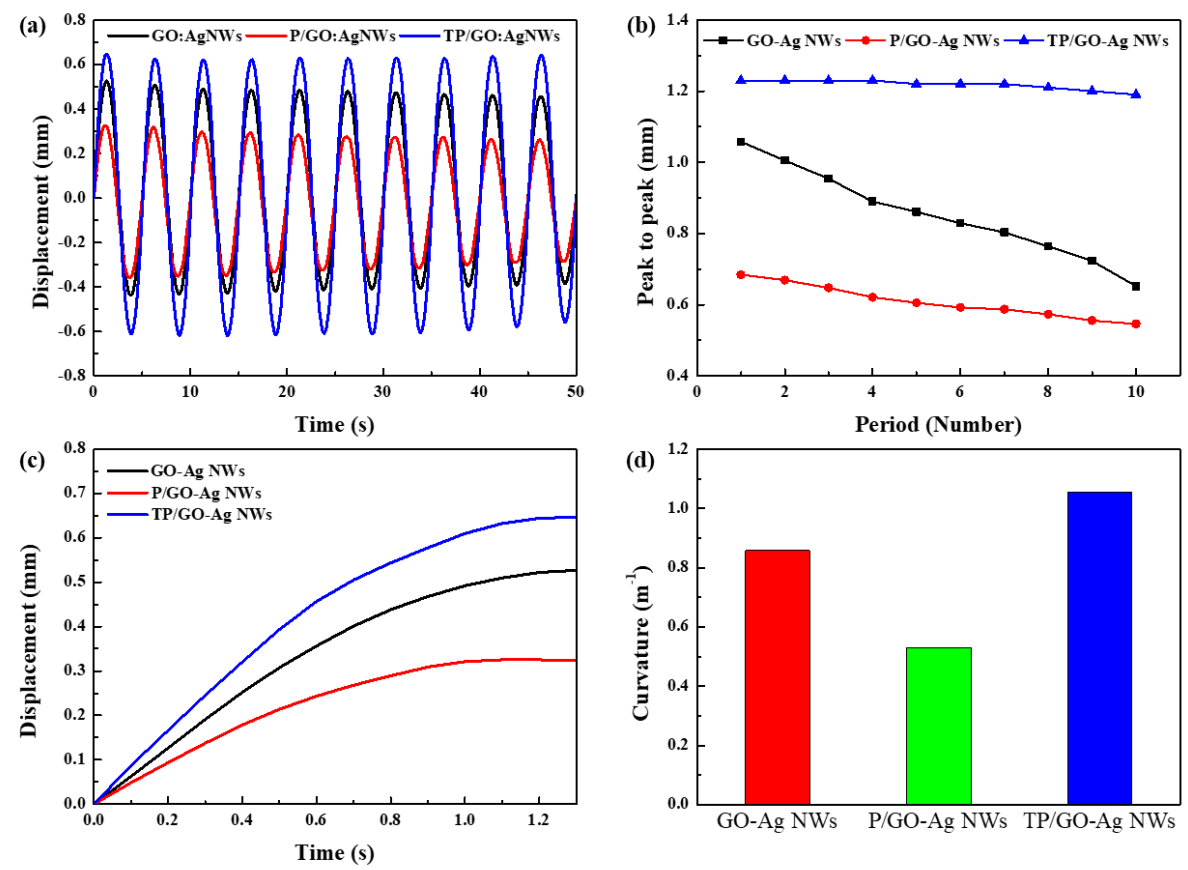

Figure 5. (a) Displacement versus time $\left( \pm 2.5 \mathrm{~V}_{\mathrm{AC}}, 0.2 \mathrm{~Hz}\right)$, (b) peak-to-peak performance, (c) response rate, and (d) bending curvature of three different ionic electroactive polymers (IEAPs) (based on GO-Ag NWs, P/GO-Ag NWs, and TP/GO-Ag NWs).

As shown in Figure 5b, the peak-to-peak performance of the TP/GO-Ag-NW-based IEAPs showed a lower slope than that of the other IEAPs, meaning that the TP/GO-Ag-NW-based IEAPs are more durable than the other IEAPs. Figure $5 \mathrm{c}$ shows the actuation performance, from $0 \mathrm{~s}$ to $1.3 \mathrm{~s}$, of a segment of the harmonic response from Figure 5a. The response rate of the TP/GO-Ag-NW-based IEAPs was 
$34.83 \%$ and $23.87 \%$ faster than those with the P/GO-Ag-NW-based IEAPs and GO-Ag-NW-based IEAPs, respectively. Figure $5 \mathrm{~d}$ shows the curvatures of the three types IEAPs. The maximum curvature of TP/GO-Ag-NW-based IEAPs was approximately $1.054 \mathrm{~m}^{-1}$, which is higher than that of the GO-Ag-NW-based IEAPs $\left(0.858 \mathrm{~m}^{-1}\right)$ and P/GO-Ag-NW-based IEAPs $\left(0.53 \mathrm{~m}^{-1}\right)$.

After measuring the actuation performance, SEM was used to observe the change in the electrode surfaces of the three types IEAPs. The decreased actuation performance of the GO-Ag-NW- and P/GO-Ag-NW-based IEAPs in Figure 5a,b can be explained by Figure 6, which shows SEM images of the electrode surface of three types of IEAPs before (Figure $6 \mathrm{a}-\mathrm{c}$ ) and after (Figure $6 \mathrm{~d}-\mathrm{f}$ ) the driving test. Because of the high contact resistance of the surface, heat is generated, which weakens metal NWs. This is a critical drawback of metal NWs networks, which may disconnect when voltage is administered to IEAPs owing to the resulting heat. Accordingly, serious transformation and network disconnection was observed for the Ag NWs in the IEAPs based on GO-Ag NWs and P/GO-Ag NWs. In contrast, the shape and network connection of the Ag NWs in the TP/GO-Ag-NW-based IEAPs were well maintained. Ultimately, this TP layer, which shows enhanced stability and durability during driving, can be used as a protective layer to decrease the high contact resistance of the GO-Ag NW electrode.
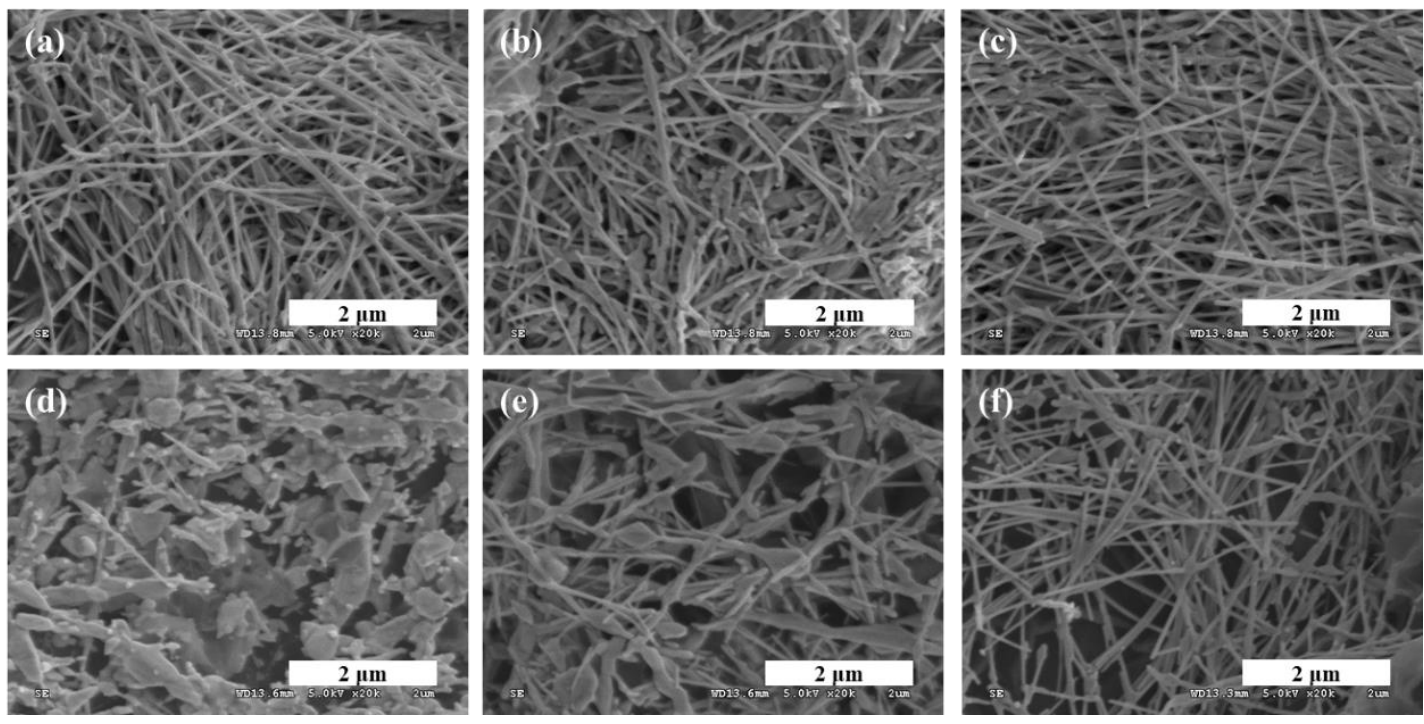

Figure 6. Surface scanning electron microscopy (SEM) images of the electrodes before (top) and after (bottom) a driving test of IEAPs based on (a,d) GO-Ag NWs, (b,e) P/GO-Ag NWs, and $(\mathbf{c}, \mathbf{f})$ TP/GO-Ag NWs.

\section{Discussion and Conclusions}

Triton X-100, a nonionic surfactant, was used to functionally enhance a PEDOT:PSS protective layer on a GO-Ag NW electrode. Triton X-100 induced the shape deformation of PEDOT:PSS, which reduced both sheet resistance and surface tension. When applied to the GO-Ag NW electrode, the PEDOT:PSS mixed with $7.5 \mathrm{wt} \%$ Triton X-100 provided the lowest sheet resistance. The optimal coating conditions for PEDOT:PSS mixed with $7.5 \mathrm{wt} \%$ Triton X-100 were $30 \mathrm{~s}$ of coating at $1000 \mathrm{rpm}$. The sheet resistance of the TP/GO-Ag NW electrode coated under these optimal conditions was $160 \mathrm{~m} \Omega / \mathrm{sq}$., which was $49.73 \%$ and $82.10 \%$ lower than those of the GO-Ag NW and pure PEDOT:PSS coated GO-Ag NWs (P/GO-Ag NWs) electrodes, respectively. The driving performance of TP/GO-Ag-NW-based IEAPs was significantly better than that of the IEAPs based on GO-Ag NWs and P/GO-Ag NWs. Furthermore, the shape and network connection of the Ag NWs in the TP/GO-Ag-NW-based IEAPs was well maintained, as revealed by SEM images. Therefore, both the stability and durability of TP/GO-Ag-NW-based IEAPs were confirmed to improve. These results demonstrate the possibility of improving electrodes with high contact resistance in terms of durability and stability. 
Author Contributions: Initial idea and writing-original draft preparation, M.P.; experiments and analysis of experiments data, S.Y.; investigation and data curation, Y.B.; experimental data curation and validation, S.K.; project administration and writing-review and editing, M.J.

Funding: This research was supported by a grant to the Bio-Mimetic Robot Research Center Funded by the Defense Acquisition Program Administration. It was also supported by the Agency for Defense Development in 2019 (UD160027ID) and the Basic Science Research Program through the National Research Foundation of Korea (NRF) funded by the Ministry of Education (NRF-2016R1D1A1B01011724).

Conflicts of Interest: The authors declare no conflict of interest.

\section{References}

1. Kim, J.; Jeon, J.H.; Kim, H.J.; Lim, H.; Oh, I.K. Durable and water-floatable ionic polymer actuator with hydrophobic and asymmetrically laser-scribed reduced graphene oxide paper electrodes. ACS Nano 2014, 8 , 2986-2997. [CrossRef] [PubMed]

2. Kim, K.; Palmre, V.; Jeon, J.H.; Oh, I.K. IPMCs as EAPs: Materials. In Electromechanically Active Polymers: A Concise Reference; Springer: Cham, Switzerland, 2016; pp. 151-170.

3. Shahinpoor, M.; Kim, K.J. Ionic polymer-metal composites: I. Fundamentals. Smart Mater. Struct. 2001, 10, 819. [CrossRef]

4. Fujiwara, N.; Asaka, K.; Nishimura, Y.; Oguro, K.; Torikai, E. Preparation of gold-solid polymer electrolyte composites as electric stimuli-responsive materials. Chem. Mater. 2000, 12, 1750-1754. [CrossRef]

5. Palmre, V.; Lust, E.; Jänes, A.; Koel, M.; Peikolainen, A.L.; Torop, J.; Johanson, U.; Aabloo, A. Electroactive polymer actuators with carbon aerogel electrodes. J. Mater. Chem. 2011, 21, 2577-2583. [CrossRef]

6. Lee, D.Y.; Park, I.S.; Lee, M.H.; Kim, K.J.; Heo, S. Ionic polymer-metal composite bending actuator loaded with multi-walled carbon nanotubes. Sens. Actuator A Phys. 2007, 133, 117-127. [CrossRef]

7. Akle, B.J.; Leo, D.J. Single-walled carbon nanotubes-Ionic polymer electroactive hybrid transducers. J. Intell. Mater. Syst. Struct. 2008, 19, 905-915. [CrossRef]

8. Akle, B.; Nawshin, S.; Leo, D. Reliability of high strain ionomeric polymer transducers fabricated using the direct assembly process. Smart Mater. Struct. 2007, 16, S256. [CrossRef]

9. Palmre, V.; Brandell, D.; Mäeorg, U.; Torop, J.; Volobujeva, O.; Punning, A.; Johanson, U.; Kruusmaa, M.; Aabloo, A. Nanoporous carbon-based electrodes for high strain ionomeric bending actuators. Smart Mater. Struct. 2009, 18, 095028. [CrossRef]

10. Fukushima, T.; Asaka, K.; Kosaka, A.; Aida, T. Fully plastic actuator through layer-by-layer casting with ionic-liquid-based bucky gel. Angew. Chem. Int. Ed. 2005, 44, 2410-2413. [CrossRef] [PubMed]

11. Akle, B.J.; Bennett, M.D.; Leo, D.J.; Wiles, K.B.; McGrath, J.E. Direct assembly process: A novel fabrication technique for large strain ionic polymer transducers. J. Mater. Sci. 2007, 42, 7031-7041. [CrossRef]

12. Yoo, S.; Park, M.; Kim, S.; Chung, P.S.; Jeon, M. Graphene Oxide-Silver Nanowires Paper Electrodes with Poly (3,4-ethylenedioxythiophene)-Poly (styrenesulfonate) to Enhance the Driving Properties of Ionic Electroactive Polymer Actuators. Nanosci. Nanotechnol. Lett. 2018, 10, 1107-1112. [CrossRef]

13. Hwang, B.; Lim, S. PEDOT: PSS Overcoating Layer for Mechanically and Chemically Stable Ag Nanowire Flexible Transparent Electrode. J. Nanomater. 2017, 1489186.

14. Yang, L.; Zhang, T.; Zhou, H.; Price, S.C.; Wiley, B.J.; You, W. Solution-processed flexible polymer solar cells with silver nanowire electrodes. ACS Appl. Mater. Interface 2011, 3, 4075-4084. [CrossRef] [PubMed]

15. Mayousse, C.; Celle, C.; Carella, A.; Simonato, J.P. Synthesis and purification of long copper nanowires. Application to high performance flexible transparent electrodes with and without PEDOT: PSS. Nano Res. 2014, 7, 315-324. [CrossRef]

16. Park, M.; Kim, J.; Song, H.; Kim, S.; Jeon, M. Fast and Stable Ionic Electroactive Polymer Actuators with PEDOT: PSS/(Graphene-Ag-Nanowires) Nanocomposite Electrodes. Sensors 2018, 18, 3126. [CrossRef] [PubMed]

17. Koley, D.; Bard, A.J. Triton X-100 concentration effects on membrane permeability of a single HeLa cell by scanning electrochemical microscopy (SECM). Proc. Natl. Acad. Sci. USA 2010, 107, 16783-16787. [CrossRef] [PubMed]

18. Tevi, T.; Saint Birch, S.W.; Thomas, S.W.; Takshi, A. Effect of Triton X-100 on the double layer capacitance and conductivity of poly (3,4-ethylenedioxythiophene): Poly (styrenesulfonate)(PEDOT: PSS) films. Synth. Met. 2014, 191, 59-65. [CrossRef] 
19. JeongáLee, S. Improved stability of transparent PEDOT: PSS/Ag nanowire hybrid electrodes by using non-ionic surfactants. Chem. Commun. 2017, 53, 8292-8295.

20. Oh, J.Y.; Shin, M.; Lee, J.B.; Ahn, J.H.; Baik, H.K.; Jeong, U. Effect of PEDOT nanofibril networks on the conductivity, flexibility, and coatability of PEDOT: PSS films. ACS Appl. Mater. Interface 2014, 6, 6954-6961. [CrossRef] [PubMed]

21. Hummers, W.S., Jr.; Offeman, R.E. Preparation of graphitic oxide. J. Am. Chem. Soc. 1958, 80, 1339. [CrossRef] 